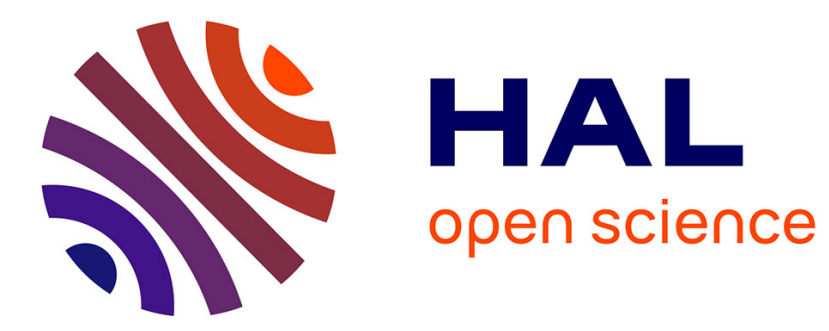

\title{
Modification of GaAs surface by low-current Townsend discharge
}

\author{
E L Gurevich, S Kittel, R Hergenröder, Yu A Astrov, L M Portsel, a N \\ Lodygin, V A Tolmachev, a V Ankudinov
}

\section{- To cite this version:}

E L Gurevich, S Kittel, R Hergenröder, Yu A Astrov, L M Portsel, et al.. Modification of GaAs surface by low-current Townsend discharge. Journal of Physics D: Applied Physics, 2010, 43 (27), pp.275302. 10.1088/0022-3727/43/27/275302 . hal-00629972

\section{HAL Id: hal-00629972 \\ https://hal.science/hal-00629972}

Submitted on 7 Oct 2011

HAL is a multi-disciplinary open access archive for the deposit and dissemination of scientific research documents, whether they are published or not. The documents may come from teaching and research institutions in France or abroad, or from public or private research centers.
L'archive ouverte pluridisciplinaire HAL, est destinée au dépôt et à la diffusion de documents scientifiques de niveau recherche, publiés ou non, émanant des établissements d'enseignement et de recherche français ou étrangers, des laboratoires publics ou privés. 


\title{
Modification of GaAs surface by low-current Townsend discharge
}

\author{
E L Gurevich $\dagger$, S Kittel $\dagger, \mathbf{R}$ Hergenröder $\dagger$, Yu A Astrov $\$$, L M

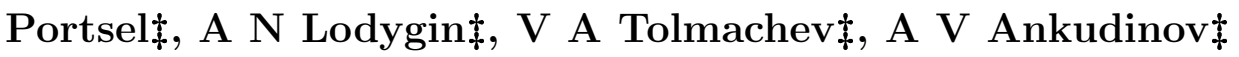 \\ $\dagger$ Leibniz-Institut für Analytische Wissenschaften - ISAS - e.V., Dortmund, 44139 \\ Germany. \\ † Ioffe Physico-Technical Institute, RAS, 194021 St.-Petersburg, Russia. \\ E-mail: evgeny.gurevich@isas.de \\ E-mail: yuri.astrov@mail.ioffe.ru
}

\begin{abstract}
Influence of stationary spatially-homogeneous Townsend discharge on the (100) surface of semi-insulating GaAs samples is studied. Samples exposed to both electrons and ions in nitrogen discharge at the current density $j=60 \mu \mathrm{A} / \mathrm{cm}^{2}$ are studied by means of X-ray photoelectron spectroscopy, ellipsometry and atomic force microscopy. It is shown that exposure to low-energy ions $(<1 \mathrm{eV})$ changes crystal structure of the semiconductor on the depth up to $10-20 \mathrm{~nm}$, although the stoichiometric composition does not change. The exposure to low-energy electrons $(<10 \mathrm{eV})$ forms an oxide layer, which is $5-10 \mathrm{~nm}$ thick. Atomic force microscopy demonstrates that the change in the surface potential of the samples may exceed $100 \mathrm{mV}$, for the both discharge polarities, while the surface roughness does not increase.
\end{abstract}

PACS numbers: 52.80.Dy, 68.47.Fg, 52.77.Bn, 68.37.Ps 


\section{Introduction}

Interaction of various materials, such as metals, semiconductors, dielectrics and polymers with gas discharge plasma leads to changes in their behaviour due to the surface modification. This phenomenon is widely used in semiconductor technology for etching, film deposition and modification of surface properties. In gas-discharge devices, however, change in the electron emission properties of cathodes under the action of gas discharge plasma may be undesirable, while it leads to instabilities of discharge parameters in time.

Changes in the semiconductor-electrode properties under interaction with gas discharge plasma is also observed, when studying semiconductorgas discharge (SGD) structures. The structure is a main part of an infrared (IR) image converter, which was invented in the beginning of $70^{\text {th }}$ [1] but similar ideas were discussed even 40 years before [2]. The device includes a thin gas discharge gap, one electrode made of a photosensitive in the IR spectral range semiconductor and the second electrode made of a glass plate covered with a transparent conductive film [3]. The device is specified by a good sensitivity and high speed of operation [4]. However, there may be observed a noticeable drift of its parameters during long operation in the continuous mode. Apparently, it is connected with change in semiconductor surface properties during the long-term action of the discharge plasma on the semiconductor electrode of the device. Such changes are observed if e.g. GaAs semiconductor wafers are used for the electrode fabrication.

The aim of the current work is to study changes in the GaAs surface, which occur after plasma treatment of the semiconductor electrodes at Townsend discharge conditions. The experiments were held in nitrogen atmosphere at low current density. The discharge effects were monitored by measuring optical properties and chemical composition of the surface by means of X-ray photoelectron spectroscopy (XPS) and

spectroscopic ellipsometry (SE) before and after the plasma treatment. Besides, roughness of the sample surface and the surface potential were studied by means of atomic force microscopy (AFM). The discharge influence was studied for both polarities of the applied feed voltage, i.e. the semiconductor surface in different experiments was treated by positive and negative (electrons and negative ions) charge carriers.

Considerable changes in the composition of the surface layer of GaAs wafers were observed at a low-current Townsend discharge even for low doses of discharge action. The discharge action was quantified as the deposited charge density, which can be calculated as product of the discharge current density and the discharge time. Oxidation of the electrode surface was found when the semiconductor electrode acted as an anode, i.e. as a result of electron irradiation. Otherwise, when semiconductor electrode served as a cathode (which corresponds to the case of ion exposure), the presence of a thin layer of structurally changed material was revealed. The AFM studies clearly demonstrated that Townsend discharge does not increase the surface roughness even for a stronger plasma treatment, while the surface potential of GaAs changes considerably. 


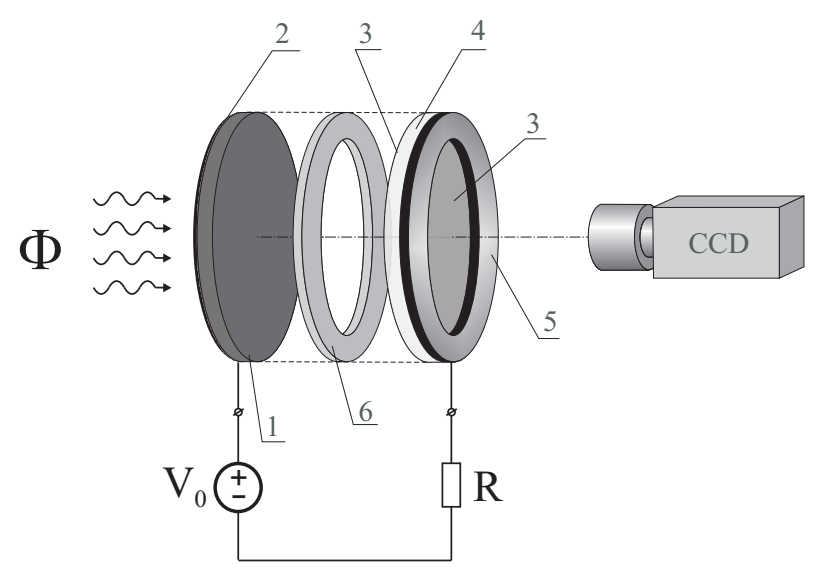

Figure 1. Experimental setup. 1 - GaAs sample; 2 - semitransparent to visible light nickel electrical contact; 3 - $\mathrm{SnO}_{2}$ layer; 4 - glass plate; 5 - copper contact ring; 6 dielectric spacer. $\Phi$ denotes visible and IR radiation illuminating the GaAs sample for the conductivity control. $R$ is a $100 \Omega$ resistor, $V_{0}$ - DC voltage source (the polarity was changed if the GaAs sample was exposed with ions).

\section{Experimental}

The experimental cell is a sandwich-like structure schematically shown in Fig. 1. The gas-discharge gap is between two electrodes, one of which is a $\mathrm{SnO}_{2}$-covered glass plate. The other electrode is a semiconductor wafer. The gas-discharge gap is formed by an opening in the dielectric spacer, placed between semiconductor wafer and the glass plate. The discharge gap width is determined by the spacer thickness, which is $90 \mu \mathrm{m}$.

The discharge influence on semiconductor wafers were studied using a semiinsulating (SI) GaAs. Such wafers are used in semiconductor industry as substrates for epitaxy. In all the experiments, the (100) surface of a GaAs sample served as a plane electrode to the gas-discharge. A semi-transparent to visible light electrical contact on a semiconductor plate was formed on the back side of the plate by means of nickel evaporation.

The experimental chamber with the gas discharge cell inside was pumped out and then filled with nitrogen at the residual pressure of $1 \times 10^{4} \mathrm{~Pa}$. Nitrogen gas was produced by evaporation of liquid nitrogen with consequent additional purification by means of a liquid-nitrogen trap. The cell was fed with high voltage by a DC power supply. The breakdown voltage of the discharge gap was approximately $300 \mathrm{~V}$.

When amplitude of the feeding voltage applied to electrodes of the device exceeds the breakdown value, discharge current flows through the hybrid SGD structure. The value of the current is controlled by resistance of the semiconductor electrode, which can be adjusted by applying homogeneous illumination from an incandescent lamp. Application of high-ohmic electrode material along with relatively small gas-discharge gap and current density provides formation of stable Townsend discharge. This discharge mode allows spatially homogeneous distribution of the discharge current density over 
the electrode plane and hence spatially homogeneous irradiation of the studied sample surface. The macroscopic homogeneity of the discharge over the discharge area was monitored by means of a CCD camera.

Electric field accelerates electrons and ions towards the electrode surfaces of a gasdischarge system. Ion interaction with the cathode is especially important, since this process is responsible for generation of secondary electrons, hence for the charge-carriers balance in the discharge. In this paper we study results of the electrode surface exposure with ions and electrons. For that, the polarity applied to the semiconductor electrode was changed in different experiments between negative (in case of its exposure with ions) and positive (used to expose the sample with electrons). Experiments were made at the discharge current density $j=60 \mu \mathrm{A} / \mathrm{cm}^{2}$. To obtain the desired current density, intensity of light illuminating the semiconductor electrode was adjusted. Such a low value of current density guarantees operation of the discharge in the Townsend mode, since it is much lower than the critical current of the Townsend-glow transition [5]. The exposure time was in the range of 15-100 minutes, so density of electric charge passed through the system during the exposure (used as a measure of the discharge effect) was in the dose range of $Q=0.05-0.50 \mathrm{C} / \mathrm{cm}^{2}$.

The chemical composition was analysed by means of X-ray photoelectron spectroscopy (XPS). This technique identifies chemical composition of studied samples according to energy spectra of photoelectrons. The electrons are emitted from corelevel shells after ionization by soft X-rays. Since the atomic states are influenced by neighbouring atoms, the XPS-technique allows identification of chemical compounds also. The used instrument was an Axis HS from Kratos Analytical, which was equipped with an ion gun for depth profiling of samples. The spectra have been recorded under the following conditions: excitation with non-monochromatic $\mathrm{MgK} \alpha$ radiation, the hemispherical analyser was operating in the fixed analyser transmission (FAT) mode with a pass energy of $80 \mathrm{eV}$ under a take-off angle of $0^{\circ}$ with respect to the surface normal. Operating in the magnetic mode, the diameter of the measured area was $150 \mu \mathrm{m}$.

For the depth profile, a beam of $\mathrm{Ar}^{+}$-ions with an energy of $3 \mathrm{keV}$ was used. Its current was $250 \mathrm{nA}$ and the angle of incidence was $45^{\circ}$ with respect to the surface normal. The sputtered area was $2 \mathrm{~mm} \times 2 \mathrm{~mm}$. The relative sputtering rate was calibrated with a $100 \mathrm{~nm} \mathrm{Ta} \mathrm{O}_{5}$ reference sample from the National Physical Laboratory. Under these conditions, the sputtering rate was $1.0 \mathrm{~nm} / \mathrm{min}$ referred to $\mathrm{Ta}_{2} \mathrm{O}_{5}$. Etching cycles of 1 minutes were chosen for in-depth profiling, which corresponds to approximately $1 \mathrm{~nm}$ per cycle.

For each sample processed in the gas discharge and for the untreated sample, a wide-scan spectrum and narrow scan spectra for O 1s, C1s, Ga3d and As 3d were acquired for each analysed depth. The carbon on the surface is due to normal surface contamination. C1s peak was strongly interfered by the Ga LMM Auger peak. Even though a contamination peak of $\mathrm{C} 1 \mathrm{~s}$ could be observed at the surface, a quantification of the $\mathrm{C} 1$ s signal under these conditions was not useful. For oxygen, the whole $\mathrm{O} 1 \mathrm{~s}$ 


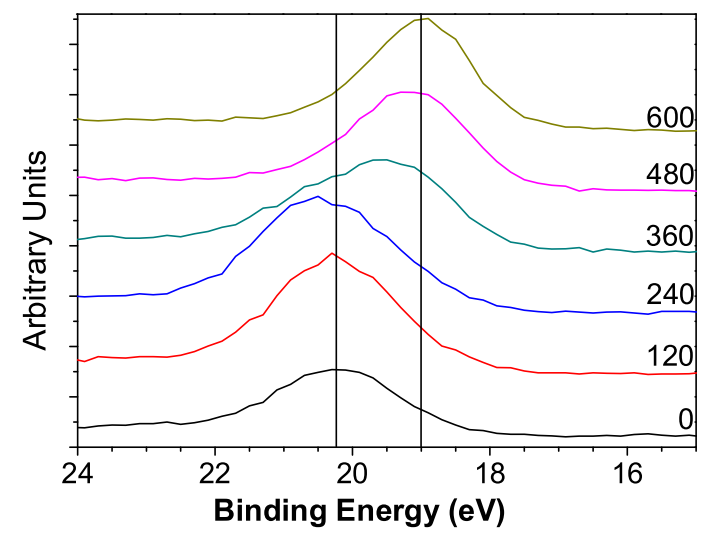

Figure 2. XPS-spectra of Ga 3d measured for irradiation dose $Q=0.23 \mathrm{C} / \mathrm{cm}^{2}$ after different sputter times. Numbers at the right side is the sputter time in seconds. The transition from oxide (binding energy $20.2 \mathrm{eV}$ ) to GaAs (binding energy $19.0 \mathrm{eV}$ ) is obvious. The semiconductor served as the positive electrode of the gas-discharge system.

signal was quantified as one peak.

Comparative narrow scan spectra for arsenic and gallium $3 \mathrm{~d}$ peaks can be found in Figures 2 and 3, respectively; curves corresponding to different depths are shifted vertically for clarity. Numbers at the curves indicate the corresponding sputtering time in seconds; the resulting in-depth step is approximately $2 \mathrm{~nm}$. In case of gallium two components were fitted: GaAs at approximately $19 \mathrm{eV}$ binding energy and $\mathrm{Ga}_{2} \mathrm{O}_{3}$ with a constant shift of $+1.2 \mathrm{eV}$. In Fig. 2 one can clearly see that near the surface the peak is located at approximately $20.2 \mathrm{eV}$, which corresponds to the gallium oxide. However the oxide peak becomes weaker and the GaAs peak at $19 \mathrm{eV}$ stronger, while analysing layers being deeper under the sample surface. A similar behaviour can be observed for arsenic in Fig. 3, where three components are detected and fitted: As bound as GaAs at $41 \mathrm{eV}$ and as oxides $\mathrm{As}_{2} \mathrm{O}_{3}$ at $44.5 \mathrm{eV}$ and, in some cases, as $\mathrm{As}_{2} \mathrm{O}_{5}$ at $46 \mathrm{eV}$ binding energies. One can see that the oxide dominates only in the surface layer, which is $6-8 \mathrm{~nm}$ deep.

Common pre-quantification procedure was applied to the measured XPS spectra: First, the individual peaks were Gaussian-fitted and then integrated after the base line subtraction. Quantification of the spectra has been done with relative sensitivity factors known from literature [6] corrected for the particular instrument used here. Due to the low binding energy of Ga $3 \mathrm{~d}$ and As $3 \mathrm{~d}$ levels, values had to be extrapolated. For this reason, the quantification has to be treated as semi-quantitative. Nevertheless the depth profiles indicate the trend of the chemical changes which are made during the irradiation with electrons or ions as a function of the depth.

For each analysed depth the chemical composition is calculated according to equation (1) below. The content $C_{i}$ of an $i^{\text {th }}$ component is proportional to the corresponding measured XPS peak area $I_{i}$ divided by the corresponding relative 


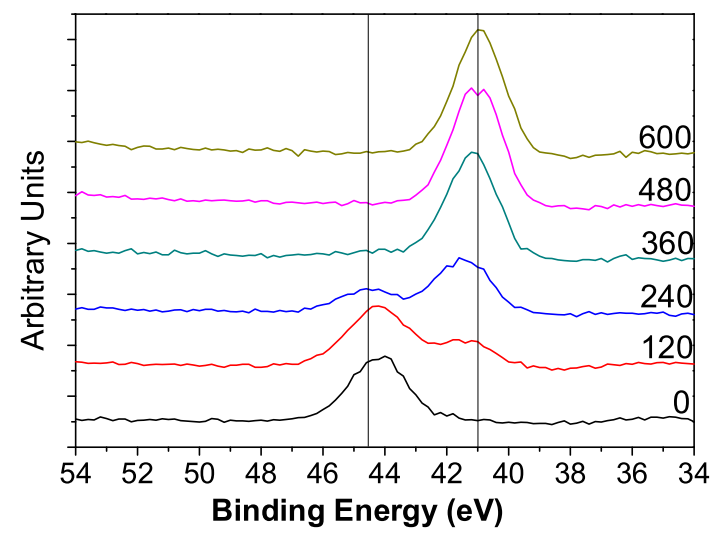

Figure 3. XPS-spectra of As 3d measured for irradiation dose $Q=0.23 \mathrm{C} / \mathrm{cm}^{2}$ after different sputter times. Numbers at the right side is the sputter time in seconds. The transition from arsenic oxide (binding energy $44.5 \mathrm{eV}$ ) to GaAs (binding energy $41 \mathrm{eV}$ ) can be proved by the chemical shift of the As $3 \mathrm{~d}$ peak. The semiconductor served as the positive electrode of the gas-discharge system.

sensitivity factor $k_{i}$. The result is normalized to the sum of all measured components:

$$
C_{i}=\frac{I_{i} / k_{i}}{\sum_{j} I_{j} / k_{j}} \times 100 \%
$$

Optical properties of wafer surface treated with the gas-discharge plasma were investigated by spectroscopic ellipsometry (SE). Ellipsometry measures change in light polarization as a result of reflection from a sample surface at a non-normal incidence. The change in polarization is given by a complex number $\rho$ related to the sample reflection properties as:

$$
\rho=\frac{R_{p}}{R_{s}}=\tan \Psi e^{i \Delta},
$$

where $R_{s}$ and $R_{p}$ are complex reflection coefficients for light waves with the electric field polarized parallel and perpendicular to the plane of incidence, while $\Psi$ and $\Delta$ are the ellipsometric parameters of the polarized light, measured in experiments. In spectroscopic ellipsometry $\rho$ is measured as a function of photon energy $h \nu$ at a fixed angle of incidence $\phi_{0}$. For convenience, $\rho$-data are sometimes converted to the complex pseudo-dielectric (PD) function, assuming a two-phase (ambient-substrate) model [7]

$$
\langle\varepsilon\rangle=\left\langle\varepsilon_{1}\right\rangle+i\left\langle\varepsilon_{2}\right\rangle=\sin ^{2} \phi_{0}\left[1+\left(\frac{1-\rho}{1+\rho}\right)^{2} \tan ^{2} \phi_{0}\right] .
$$

The spectroscopic ellipsometer Ellipse 1891 developed in the Institute of Semiconductor physics of Siberian branch of Russian Academy of Sciences was used in the experiments. Measurements were performed at the angle of light incidence $\phi_{0}=70^{\circ}$ over the photon energy range of $1.5-5.0 \mathrm{eV}$. The thickness of the modified surface layer $d$ and information about the layer composition were obtained by fitting the measured data to model calculations. A multilayer model and the Bruggeman 
effective medium approximation (EMA) [8] are used. Selected model parameters are then varied (using the Levenburg-Marquardt algorithm) to minimize the error function. Two EMA models based on the XPS data have been used for cases of electrons and ions exposures, which will be considered in detail in corresponding Sections.

The reference data for the PD function of crystalline c-GaAs, amorphous a-GaAs and also for voids (empty spaces in a layer) were supplied together with the ellipsometer software. The GaAs native oxide and $\mathrm{Ga}_{2} \mathrm{O}_{3}$ oxide data were taken from $[9,10]$, respectively.

Electrodes of a gas-discharge system are known to be a subject of erosion under influence of ions and other highly-reactive discharge products, which lead to degradation of initially smooth electrode surface. Therefore, in the frame of the present investigation, it was of interest to track, whether the significant irradiation dose leads to deterioration of the surface of a semiconductor electrode.

A sample surface was inspected by means of optical microscope OPTEM with Mitutoyo objective (with lateral optical resolution of $1 \mu \mathrm{m}$ ) and atomic force microscope (AFM) INTEGRA Aura produced by NT-MDT company. The tapping mode with the average interaction force less than $1 \mathrm{nN}$ and contact mode with average interaction force less than $100 \mathrm{nN}$ were used for the surface profiling. Probes NSG01 and HA_NC with the tip curvature radius of less than $35 \mathrm{~nm}$, resonance frequencies of 150 and $120 \mathrm{kHz}$ and force constants of 5 and $3.5 \mathrm{~N} / \mathrm{m}$, respectively, were used. The contact potential difference (CPD) distributions over a sample surface were measured by scanning two pass Kelvin probe microscopy (SKPM) mode. In the first pass, the surface topology was measured in the tapping mode. In the second pass the probe was lifted above the surface and repeated the recorded profile simultaneously measuring the CPD signal value between the AFM probe and the sample surface.

\section{Experimental results}

\subsection{Exposure with electrons}

In-depth profiles of chemical composition obtained with the XPS technique for an unprocessed GaAs sample are represented by Fig. 4. The data show relative concentration of gallium arsenide, gallium covalently bound to oxygen as well as arsenic bound to oxygen and for oxygen independent on the chemical bounds. All graphs are normalized to the sum of all measured components according to the equation (1), and the results are shown in atomic percent. One can see that the natural oxide layer (mixture $\mathrm{Ga}_{2} \mathrm{O}_{3}$ and $\mathrm{As}_{2} \mathrm{O}_{3}$ ) is present in the surface layer of the unprocessed sample. However, it is extremely thin: the thickness of the native oxide layer does not exceed one nanometer, while $\mathrm{As}_{2} \mathrm{O}_{5}$ was not detected.

Figure 5 shows the concentration profiles for the surface layer of a sample exposed with electrons. The data refer to the exposure $Q=0.23 \mathrm{C} / \mathrm{cm}^{2}$. We also measured gallium and arsenic separately: The relative atomic concentrations of $\mathrm{Ga}$ and $\mathrm{As}$ in the 


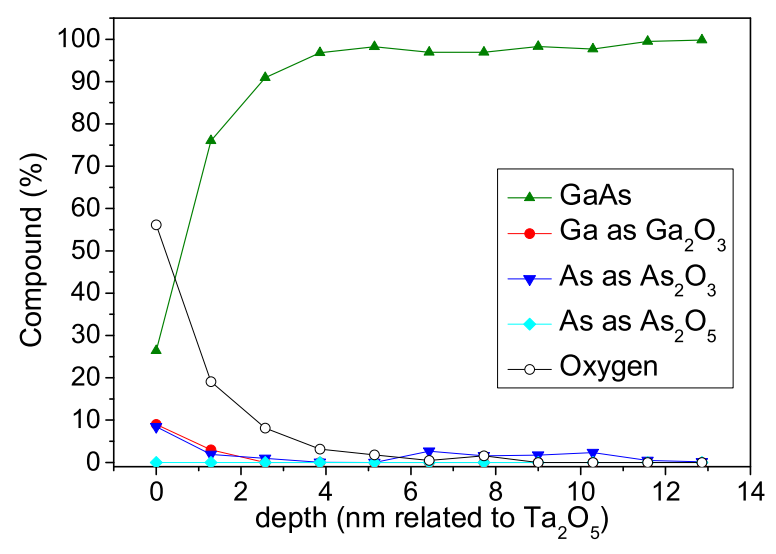

Figure 4. XPS in-depth profiles of composition for unprocessed GaAs sample.

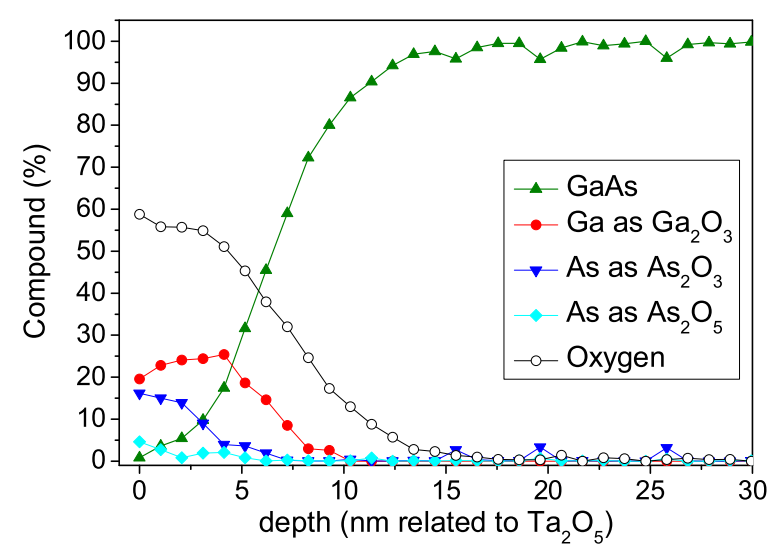

Figure 5. XPS in-depth profiles of composition for GaAs sample irradiated by electrons with dose of $0.23 \mathrm{C} / \mathrm{cm}^{2}$.

sample bulk were close to $50 \%$, which agrees with natural content of these elements in a GaAs crystal. Approaching the semiconductor surface, the concentration of GaAs decreases due to presence of the oxide film. Thickness of $\mathrm{Ga}_{2} \mathrm{O}_{3}$ layer is about $5-7 \mathrm{~nm}$. For the content of $\mathrm{As}_{2} \mathrm{O}_{3}$, a similar dependence is observed, whereas the $\mathrm{As}_{2} \mathrm{O}_{5}$ content is negligibly small.

To investigate optical properties of GaAs as dependent on the electron irradiation dose, a set of samples exposed with doses in the range of $0.05-0.30 \mathrm{C} / \mathrm{cm}^{2}$ was prepared. The result of their study with the spectroscopic ellipsometry technique is represented in Fig. 6, where the imaginary part of the $\mathrm{PD}$ function spectrum $\left\langle\varepsilon_{2}\right\rangle$ is shown for different doses of exposure. The data refer to an untreated sample (a) and samples exposed by electrons with doses $Q=0.15$ and $0.3 \mathrm{C} / \mathrm{cm}^{2}$, (b) and (c), correspondingly. The reference data for the imaginary part of PD function of crystalline GaAs is also shown.

The pseudo-dielectric function of a crystalline material is directly related to its electronic energy-band structure. So, the features observed in the $\left\langle\varepsilon_{2}\right\rangle$ spectra are 


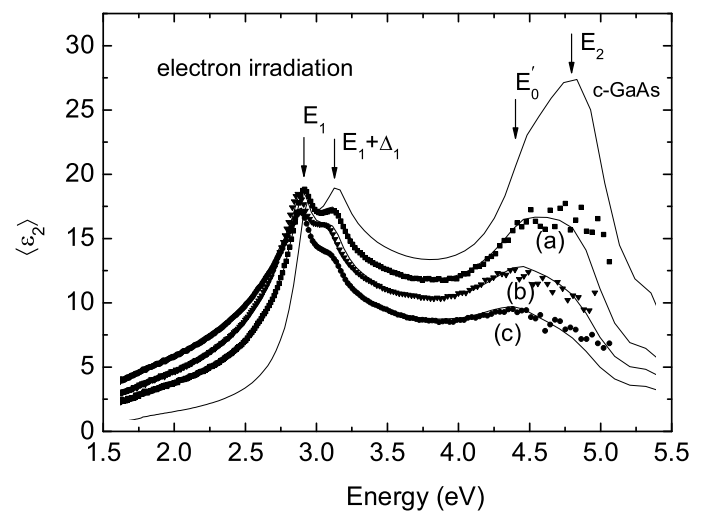

Figure 6. Imaginary part of pseudo-dielectric function spectrum obtained from SE data for samples irradiated with electrons with different doses $Q$. (a) untreated sample; (b) $Q=0.15 \mathrm{C} / \mathrm{cm}^{2}$; (c) $Q=0.3 \mathrm{C} / \mathrm{cm}^{2}$.

attributed to interband critical points of GaAs [11, 12]. As seen in Fig. 6, the ellipsometric spectra contain peaks at $E_{1}, E_{1}+\Delta_{1}(h \nu=2.91$ and $3.14 \mathrm{eV}), E_{0}^{\prime}(4.45 \mathrm{eV})$ and $E_{2}(4.77 \mathrm{eV})$ interband transitions, while contribution of other transitions such as $E_{0}$ and $E_{0}+\Delta_{0}(h \nu=1.42,1.75 \mathrm{eV})$ is weak and not observed clearly.

The most significant changes in the PD function spectrum for exposed samples take place in the $4.0-5.0 \mathrm{eV}$ range. The amplitudes of $E_{0}^{\prime}$ and $E_{2}$ peaks become smaller compared to the case of the untreated surface, while the amplitude of the $E_{1}$ and $E_{1}+\Delta_{1}$ doublet do not change significantly. The higher the electron exposure dose, the lower are the $E_{0}^{\prime}$ and $E_{2}$ peaks amplitudes (data (b), (c)).

To obtain information about the chemical composition and structure of surface layers from SE data, a multilayer model was used. Usually, complicated structure of a surface layer, including its roughness and complexity in composition, is described with the effective medium approximation (EMA) model [8]. According to this approach, a real surface layer is replaced by a layer having a so-called effective medium dielectric function [7].

We point out that the $\left\langle\varepsilon_{2}\right\rangle$ spectrum for the untreated sample (data (a)) is somewhat different from the corresponding reference spectrum of c-GaAs: namely, the amplitude of the peak at $4.75 \mathrm{eV}$ is reduced essentially as compared to the c-GaAs curve. A natural suggestion could be that such a discrepancy is due to presence of both an oxide film on surface of the untreated sample, and of the transition layer between oxide and bulk semiconductor. (In this relation, see Fig. 4.)

An appropriate model for the analysis of ellipsometric data for a set of samples irradiated with electrons (including the untreated one) can be formulated on the base of XPS results. The oxide $\mathrm{Ga}_{2} \mathrm{O}_{3}$ layer was found to appear on the GaAs substrate as a result of exposure with electrons (Fig. 5). This oxide is known [10] to have a larger 


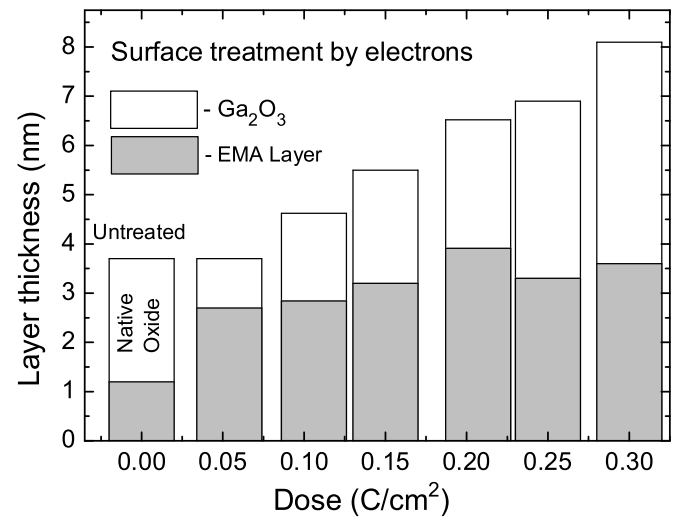

Figure 7. Thickness of $\mathrm{Ga}_{2} \mathrm{O}_{3}$ oxide and EMA layer as a function of irradiation dose with electrons.

refractive index compared to the native oxide [9], which consists typically of $\mathrm{GaAsO}_{3}$ compound. So, to interpret experimental SE data for a set of samples irradiated with electrons, we developed a model that included GaAs substrate, the effective medium layer (which is a mixture of c-GaAs and the native oxide) and a thin film of native oxide.

The spectrum of $\left\langle\varepsilon_{2}\right\rangle$ calculated for best-fitting parameters for an untreated sample is shown by solid line (a) in Fig. 6. Thus, the EMA layer of the thickness of $1.2 \mathrm{~nm}$ had a composition of $36.8 \%$ of GaAs and $63.2 \%$ of native oxide. The thickness of the native oxide film was evaluated to be $2.5 \mathrm{~nm}$. (The oxide $\mathrm{As}_{2} \mathrm{O}_{3}$ is not included into the model since its relative fraction is less than $\mathrm{Ga}_{2} \mathrm{O}_{3}$ according to the XPS measurements.)

Spectra of the imaginary part of the PD function calculated within such a model for samples exposed with different doses are shown by solid lines in Fig. 6. Apparently, there is a good correspondence of calculated and the experimental results. Parameters of the model applied in calculations are indicated in Table 1.

Results of fitting the calculated data to experimental curves, which are obtained for samples with different irradiation doses are summarized in Table 1. It shows that composition and thickness of the EMA layer depend on the irradiation dose: When it increases, the thickness of the oxide layer $\mathrm{Ga}_{2} \mathrm{O}_{3}$ grows. It reaches $4.5 \mathrm{~nm}$ at the maximal dose what is close to the data of Fig. 5. Figure 7 shows results obtained for the thickness of $\mathrm{Ga}_{2} \mathrm{O}_{3}$ and EMA layers as a function of dose.

\subsection{Exposure with ions}

As noticed in Section 2, exposure of the samples with ions was carried out in the discharge system, when the semiconductor electrode operated at the negative polarity of the applied voltage. The XPS in-depth profiles of chemical composition for a sample 
Table 1. Best-fitting parameters of the model used in processing ellipsometric data for samples irradiated by electrons.

\begin{tabular}{|c|c|c|c|c|}
\hline \multirow{2}{*}{$\begin{array}{c}\text { dose, } \\
\mathrm{C} / \mathrm{cm}^{2}\end{array}$} & \multicolumn{3}{|c|}{ EMA layer } & $\mathrm{Ga}_{2} \mathrm{O}_{3}$ layer \\
\cline { 2 - 5 } & $\mathrm{GaAs}, \%$ & $\mathrm{Ga}_{2} \mathrm{O}_{3}, \%$ & thickness, $\mathrm{nm}$ & thickness, $\mathrm{nm}$ \\
\hline \hline 0.05 & 32.7 & 67.3 & $\overline{2.7}$ & 1.0 \\
\hline 0.11 & 31.5 & 68.5 & 2.8 & 1.8 \\
\hline 0.15 & 25.3 & 74.7 & 3.2 & 2.3 \\
\hline 0.21 & 29.9 & 70.1 & 3.9 & 2.6 \\
\hline 0.26 & 24.1 & 75.9 & 3.3 & 3.6 \\
\hline 0.30 & 23 & 77 & 3.6 & 4.5 \\
\hline
\end{tabular}

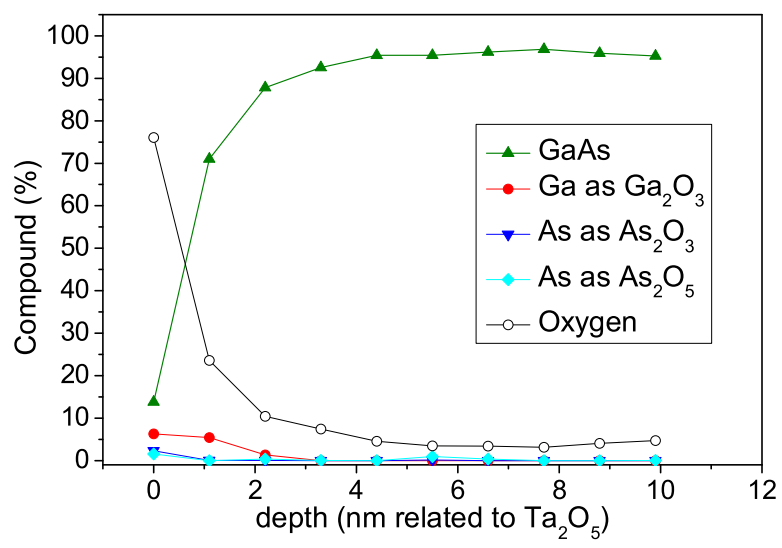

Figure 8. XPS in-depth profiles of composition for a sample irradiated by ions with the dose of $0.47 \mathrm{C} / \mathrm{cm}^{2}$.

subjected to the exposure by nitrogen ions with the maximal dose $Q=0.47 \mathrm{C} / \mathrm{cm}^{2}$ are represented by Fig. 8. The data show that there is no significant influence of irradiation with ions on chemical composition of a near-surface layer as compared to the untreated sample (Fig. 4).

On the other hand, ellipsometric data demonstrate essential difference in optical properties of the untreated and exposed surfaces of the semiconductor. Figure 9 shows the imaginary part of PD function spectra $\left\langle\varepsilon_{2}\right\rangle$ for samples exposed with ions at different doses. Data (a) are obtained for untreated surface and are the same as in Fig. 6. Data (b) and (c) correspond to spectra for surfaces exposed with ions of doses 0.23 and $0.47 \mathrm{C} / \mathrm{cm}^{2}$. One can see that there is a noticeable change in the $\left\langle\varepsilon_{2}\right\rangle$ spectrum at an increase in the irradiation dose: The significant decrease in the spectra maxima and a slight red shift of the $E_{1}, E_{1}+\Delta_{1}$ doublet are observed, while the $E_{2}$ pike amplitude decreases also (cf. Fig. 6). When comparing these results with the corresponding data for the electron-exposed samples, one can see the essential difference in the behaviour of the PD function spectra for the two cases.

It is worth noting that similar changes in spectra of the PD function of GaAs 


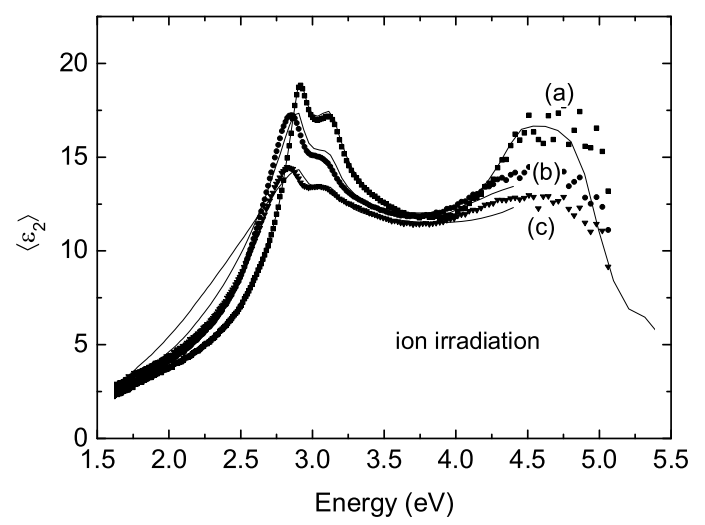

Figure 9. Imaginary part of the PD function spectrum obtained from SE data for GaAs samples irradiated by ions with different doses $Q$. (a) untreated sample; (b) $Q=0.23 \mathrm{C} / \mathrm{cm}^{2}$; (c) $Q=0.47 \mathrm{C} / \mathrm{cm}^{2}$. Points are experimental data, continuous curves calculation with the EMA model.

samples are observed when the semiconductor surface is processed by ions during electron cyclotron resonance plasma etching [13], or at a low-energy hydrogen ion bombardment [14]. The ion implantation of elements into GaAs is known to be also accompanied by changes in the optical properties of semiconductor [15]. These effects are related to a thin film of damaged crystal structure, which is formed on the surface under ion bombardment. When an energetic ion penetrates a solid target, lattice atoms displacement occurs due to collisions between the incident ions and the near-surface atoms. In semiconductors, such displacement processes cause accumulation of structural defects within the lattice. Hence, an amorphous surface layer may be built up.

As mentioned above, XPS measurements have not revealed essential changes in the chemical composition of the semiconductor due to its irradiation with ions. Therefore, we assume from the aforesaid that ion bombardment causes in our experiments only structural changes of the semiconductor surface, leaving the chemical composition of semiconductor material almost unchanged.

A usual way of describing optical response of such a material is to assume that the surface layer of the semiconductor is a physical mixture of crystalline and amorphous regions, and to use the EMA method to calculate the resulting PD function of modified GaAs [16]. However, in spite of its physical simplicity, the EMA model is not always able to describe the behaviour of PD function spectrum properly in the overall range of transition from the crystalline material to amorphous. As the authors of [15] have demonstrated, a better agreement between experimental and theoretical results can be achieved in the harmonic oscillator approximation (HOA), where the PD function is represented as a superposition of harmonic oscillators.

Nevertheless, as a first approximation to analyse the effect of irradiation of GaAs 


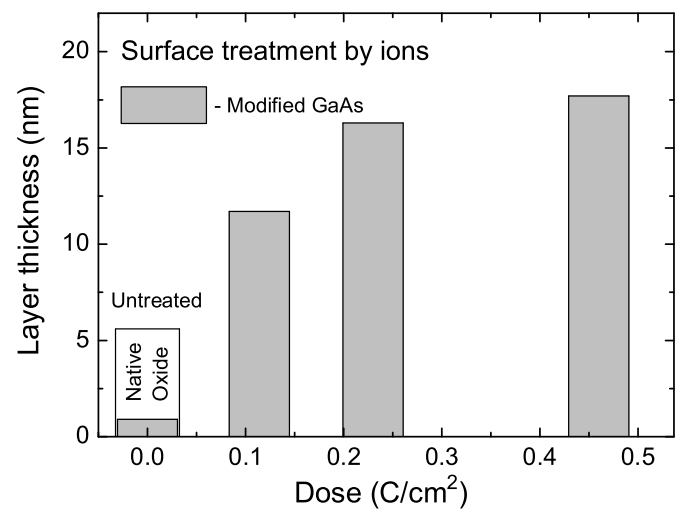

Figure 10. Thickness of the modified GaAs layer as a function of irradiation dose with ions. The EMA model of the modified GaAs layer consists of a mixture of crystalline c-GaAs, amorphous a-GaAs and voids, see Table 2.

surface with ions, we restricted our consideration to the EMA model. The physical model consisted from the bulk semiconductor substrate combined with a modified GaAs layer. We did not include the oxide layer in the model, because the XPS measurements had not revealed the appearance of the oxide due to exposure with ions. The modified GaAs layer is assumed to be the mixture of crystalline c-GaAs, amorphous a-GaAs and voids. The spectra of imaginary part of PD function calculated with this model are shown by solid lines in Fig. 9 (b), (c), see the corresponding best-fitting parameters for these curves in Table 2.

One can see that the applied EMA model describes main features of spectra modification at the exposure with ions: A decrease in amplitude for both the doublet structure at photon energies near $3 \mathrm{eV}$, and of the pike at $\sim 5 \mathrm{eV}$. However, there are observed discrepancies between the results of the model and experimental data. At first, an expressed difference exists between experimental and theoretical curves in the range of low photon energy 1.5-2.5 eV. Furthermore, the model does not take into account the experimentally observed slight red shift of $E_{1}, E_{1}+\Delta_{1}$ doublet. As it has been pointed out above, more sophisticated models - like the harmonic oscillators approximation should be used for a better consistency in theoretical description of the PD function spectra.

Parameters of modified surface model obtained for samples exposed by ions with doses in the range of $Q=0.11-0.47 \mathrm{C} / \mathrm{cm}^{2}$ are summarized in Table 2. According to these data, the ion irradiation leads to formation of structurally damaged layer on the semiconductor surface. It is interesting to note that the experimental data can be described in the approximation, where the composition of this layer does not depend on the irradiation dose and is approximately: c-GaAs 37\%; a-GaAs 44\%; voids 19\%. However, the thickness of the layer increases with $Q$, and the maximal measured 
Table 2. Best-fitting parameters of the model used in processing ellipsometric data for samples irradiated by ions

\begin{tabular}{|c|c|c|c|c|}
\hline \multirow{2}{*}{$\begin{array}{c}\text { dose }, \\
\mathrm{C} / \mathrm{cm}^{2}\end{array}$} & \multicolumn{4}{|c|}{ composition of the modified layer } \\
\cline { 2 - 5 } & $\mathrm{c}-\mathrm{GaAs}, \%$ & a-GaAs, $\%$ & voids, $\%$ & thickness, $\mathrm{nm}$ \\
\hline \hline 0.11 & 34 & 46 & 20 & 11.7 \\
\hline 0.23 & 36.8 & 44.2 & 19 & 16.3 \\
\hline 0.47 & 36.8 & 44.2 & 19 & 17.7 \\
\hline
\end{tabular}

thickness is $\sim 18 \mathrm{~nm}$. The variation of the layer thickness, which is modified with the ions exposure, is shown in Fig. 10 for different exposure doses. The data for the untreated surface is shown in the figure also.

We point out that colour of the sample surface changes after processing by large doses of particle flows at both discharge polarities. However, no erosion of the semiconductor surface was detected by means of optical microscopy. Further investigation of the surface quality of the samples was done with the atomic force microscopy.

\subsection{AFM analysis of the semiconductor electrode surface.}

An example of the AFM image of the surface exposed by electrons with dose of $0.37 \mathrm{C} / \mathrm{cm}^{2}$ is shown in Fig. 11. The scanning area is $1 \mu \mathrm{m} \times 1 \mu \mathrm{m}$. The average height of surface irregularities is about $1.5 \mathrm{~nm}$; the distributions of the heights relatively to the average value is plotted at the bottom of the figure for the untreated sample (curve 1), and samples exposed with ions (2) and with electrons (3). These data clearly demonstrate that there is no significant difference between these distributions (the width variation is less than $6 \AA$ ). Increasing the scanned area to $10 \mu \mathrm{m} \times 10 \mu \mathrm{m}$ is accompanied by an increase of the characteristic width of height distribution; however, this effect is relatively small. We can conclude, therefore, that the discharge plasma treatment with the doses used in the experiments does not cause any noticeable erosion of the GaAs surface.

The contact potential difference (CPD) distribution over the surface area of the sample exposed by electrons with the dose of $0.37 \mathrm{C} / \mathrm{cm}^{2}$ is shown in Fig. 12. The measurements were peformed in the two-pass mode (see Section 2). The distance between the Kelvin probe and semiconductor surface was $50 \mathrm{~nm}$. The scanned area of $100 \mu \mathrm{m} \times 100 \mu \mathrm{m}$ was chosen in such a way that both untreated and exposed parts of the sample surface were investigated. The left side of the figure corresponds to the untreated surface. It is seen that there is a difference between CPD signals in both areas, which is about $300 \mathrm{mV}$. The CPD signal profile averaged over the area outlined by the white rectangle in the top part of the figure is plotted in the graph below. We note that the observed effect corresponds to the bending down the surface electron band of GaAs (at approximately $300 \mathrm{mV}$ ) compared to the initial state of the semiconductor. 


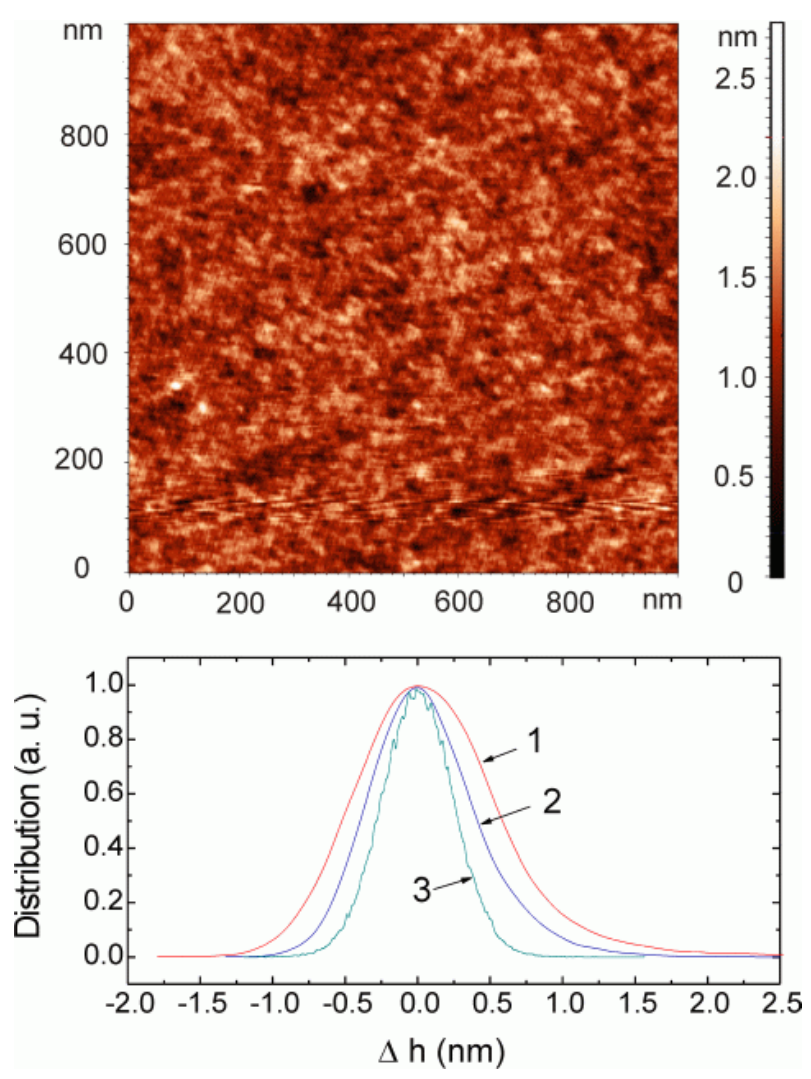

Figure 11. Upper panel: AFM image of the surface exposed by electrons with dose of $0.37 \mathrm{C} / \mathrm{cm}^{2}$, see the text. Shown at the lower panel are distributions of amplitudes of surface irregularities for: (1) untreated surface; (2) surface exposed by ions with dose of $0.37 \mathrm{C} / \mathrm{cm}^{2} ;(3)$ surface exposed by electrons with dose of $0.37 \mathrm{C} / \mathrm{cm}^{2} . \Delta h$ is the height deviation relative to the average value.

Similar results were obtained for the GaAs surface exposed with ions. At a comparable dose of the exposure, the absolute value of the CPD signal was approximately two times lower than for the exposition with electrons, while the sign of the effect is opposite.

\section{Conclusion}

Our results demonstrate that properties of a GaAs electrode are changed in the Townsend discharge due to exposure to the charge-carrier flux. The effect is observed while the semiconductor is exposed for both electrons and ions even for low current densities. The exposure of GaAs to electron flux stimulates growth of an oxide film on the semiconductor surface. There may be two possible sources of oxygen needed for the oxide growth in the gas-discharge environment: (i) Oxygen could be released from the $\mathrm{SnO}_{2}$ film (which served as the second electrode to the discharge plasma) by its degradation due to exposure to active discharge products; (ii) It may be present as an impurity of a tiny content in nitrogen. We note that the oxide film also grows if pure 


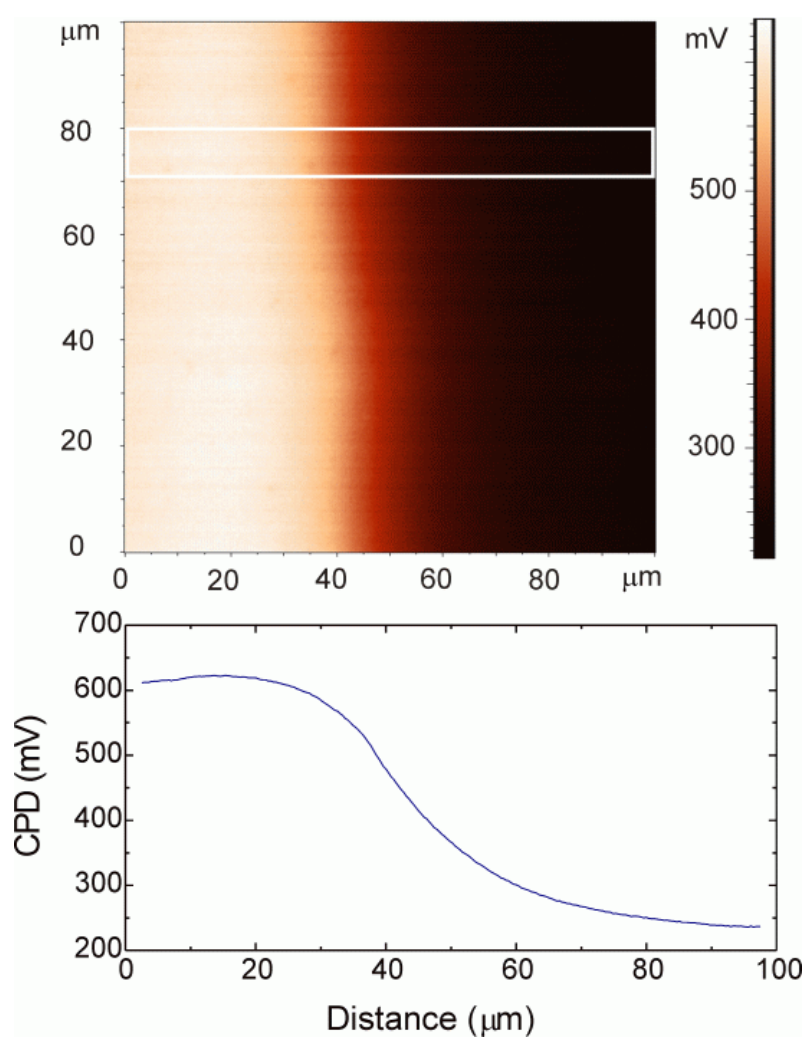

Figure 12. Map of surface potential (upper part) and the potential profile for a sample exposed by electrons at dose of $0.37 \mathrm{C} / \mathrm{cm}^{2}$. The exposed part of the sample (right) is characterized by a lower potential.

(99.997\%) nitrogen was used instead of nitrogen evaporated from liquid state.

Concerning the observed electron-induced acceleration of oxide growth on GaAs surface we note that in [17] oxide growth on silicon surfaces in presence of free electrons was reported. Although experiments in [17] were done for a different sample material and at different experimental conditions, we note that same processes (e.g. electron attachment to oxygen [5]) may also activate the anode oxidation in our experiments.

When semiconductor serves as the cathode to the low-current Townsend discharge, no changes in the chemical composition of the semiconductor surface was detected with the XPS method. However, these data, together with results of spectroscopic ellipsometry measurements and measurements of surface potential with the AFM scanning Kelvin probe technique reveal essential structural changes in the GaAs surface induced by ion exposure. The results obtained give an evidence of partial amorphization of the GaAs surface layer, which thickness increases with the dose of ions.

We note that the average kinetic energy of ions impacting the cathode is rather low in our experiments and does not exceed several tenths of $\mathrm{eV}$, see [18] for reference. The neutralization of such low-energy ions on a surface of metals and semiconductors is known to occur via the Auger neutralization process [19]: This occurs via tunnelling of electron from a deep electronic state of a solid to the ground state of ion that 
approaches its surface. The energy released at such a process, is transferred to the electronic subsystem of semiconductor. The amount of the transferred energy is rather large: It is in the order of ionization potential of the gas atom/molecule. In the course of relaxation of such excitations in the crystal, structural defects in the semiconductor lattice may be created near the semiconductor surface and accumulated there at subsequent ion neutralization events. It is essential that this mechanism of excitation of a semiconductor remains effective even at quite a low kinetic energy of ions bombarding the semiconductor surface.

In our experiments, plasma treatment of GaAs changes the surface potential of semiconductor, which should influence the electron work function and, correspondingly, the secondary electron emission coefficient $\gamma$ [5]. This effect may obviously be a reason for the drift of parameters of microdischarge devices (in which a semiconductor electrode is used) in time.

We also note, finally, that controllable modification of a semiconductor properties by a low-current Townsend discharge may be a practically interesting technological application of microdischarges.

\section{Acknowledgement}

The work is partly supported by the President Grant of Russia for Scientific Schools (Grant 3306.2010.2), Russian Foundation for Basic Research (Grant 07-2-00168-a) and Program of fundamental research of Russian Academy of Sciences (Program No. 27).

[1] Kasymov Sh S and Paritskii L G 1973 Device for tracking images Russian Authors' certificate 1798020/18-10

[2] Lengnick T C 1933 Discharge Tube United States Patent Office, Patent 1936514

[3] Portsel L M, Astrov Yu A, Reimann I, Ammelt E and Purwins H-G 1999 J. Appl. Phys. 85 3960-65

[4] Marchenko V M, Matern S, Purwins H-G, Astrov Yu A and Portsel L M 2003 Proc. SPIE 494898

[5] Raizer Yu P 1991 Gas Discharge Physics (Berlin: Springer)

[6] Briggs D and Seah M P 1996 Practical Surface Analysis, Auger and X-ray Photoelectron Spectroscopy (John Wiley \& Sons)

[7] Azzam R M A and Bashara N M 1977 Ellipsometry and Polarized Light (Amsterdam-New YorkOxford: North Holland)

[8] Bruggeman D A G 1935 Ann. Phys. 416 636-664

[9] Zollner S 1993 Appl. Phys. Lett. 63 2523-24

[10] Rebien M, Henrion W, Hong M, Mannaerts J P and Fleischer M 2002 Appl. Phys. Lett. 81 250-52

[11] Lautenschlager P, Garriga M, Logothetidis S and Cardona M 1987 Phys. Rev. B 35 9174-88

[12] Ozaki S and Adachi S 1995 J. Appl. Phys. 78 3380-86

[13] Snyder P G, Ianno N J, Wigert B, Pittal S, Johs B and Woollam J A 1995 J. Vac. Sci. and Technol. B: Microelectronics and Nanometer Structures 13 2255-59

[14] Schubert E, Razek N, Frost F, Schindler A and Rauschenbach B 2005 J. Appl. Phys. 97023511

[15] Erman M, Theeten J B, Chambon P, Kelso S. M and Aspnes D E 1984 J. Appl. Phys. 56 2664-71

[16] Adachi S 1991 J. Appl. Phys. 69 7768-73

[17] Young E M 1988 Appl. Phys. A: Mater. Sci.\& Process. 47 259-69

[18] Brown S C 1959 Basic Data of Plasma Physics (New York: The Technology Press MIT)

[19] Hagstrum H D 1961 Phys. Rev. 12283 